\title{
2D Melting: From Liquid-Hexatic Coexistence to Continuous Transitions
}

\author{
Sebastian C. Kapfer* and Werner Krauth ${ }^{\dagger}$ \\ Laboratoire de Physique Statistique, Ecole Normale Supérieure, UPMC, \\ Université Paris Diderot, CNRS, 24 rue Lhomond, 75005 Paris, France
}

(Dated: January 8, 2015)

\begin{abstract}
The phase diagram of two-dimensional continuous particle systems is studied using Event-Chain Monte Carlo. For soft disks with repulsive power-law interactions $\propto r^{-n}$ with $n \gtrsim 6$, the recently established hard-disk melting scenario $(n \rightarrow \infty)$ holds: a first-order liquid-hexatic and a continuous hexatic-solid transition are identified. Close to $n=6$, the coexisting liquid exhibits very long orientational correlations, and positional correlations in the hexatic are extremely short. For $n \lesssim 6$, the liquid-hexatic transition is continuous, with correlations consistent with the KosterlitzThouless-Halperin-Nelson-Yong (KTHNY) scenario. To illustrate the generality of these results, we demonstrate that Yukawa particles likewise may follow either the KTHNY or the hard-disk melting scenario, depending on the Debye-Hückel screening length as well as on the temperature.
\end{abstract}

Two-dimensional particle systems with short-range interactions may form solids [1, but cannot acquire longrange positional order [2]. Rather, two-dimensional solids are characterized by long-range orientational and quasilong-range positional order, so that positional correlation functions decay algebraically. In the liquid phase, both orientational and positional order are short-ranged, and the corresponding correlation functions decay exponentially. An intermediate hexatic phase may also exist [3]. It is characterized by short-range positional and quasilong-range orientational order.

Within the Kosterlitz-Thouless-Halperin-NelsonYoung (KTHNY) theory of two-dimensional melting 3, these two symmetry-breaking transitions arise from the subsequent unbinding of topological defects: In the solid, dislocations are bound in pairs, whereas in the hexatic, free dislocations may exist. The dislocations then decompose into free disclinations which break orientational order and yield the isotropic liquid. Both phase transitions are of the continuous KosterlitzThouless type, although a first-order liquid-hexatic transition remains possible within the KTHNY framework. Alternative theories of two-dimensional melting propose a conventional first-order liquid-solid transition, in the absence of a hexatic phase 4, 5. These scenarios commonly involve the condensation of defects into grain boundaries and related aggregates [6] 8 .

Over the decades, it has been extremely difficult to decide, from theory, simulation or experiments, which of the above melting scenarios applied to specific twodimensional models. It was established only very recently that the fundamental hard-disk model has a continuous solid-hexatic transition but a first-order hexaticliquid transition [9]. This scenario continues to apply for three-dimensional hard spheres tightly confined between parallel plates [10]. Indications for such a scenario were also found for two-dimensional Yukawa particles [11].

Experimentally, evidence for liquid-hexatic coexistence was reported both for sterically stabilized uncharged colloids 12 and for charged colloids [13. In complex plas-
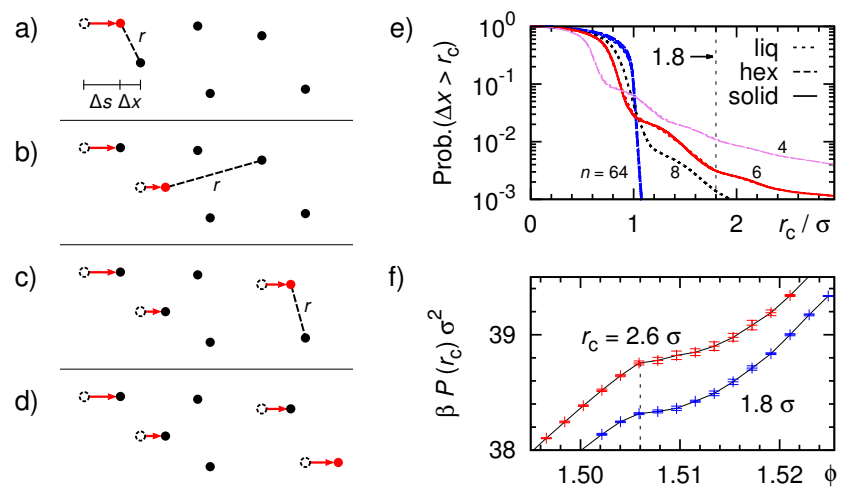

FIG. 1. (Color online) Event-Chain algorithm for continuous pair interactions. a-d) Evolution of the algorithm for four particles through three subsequent collision events. e) Probability for soft disks that a collision event takes place at a distance $r$ larger than the cutoff $r_{\mathrm{c}}$ The vertical dashed line is the cutoff chosen in this work. f) Equation of state with different cutoffs $(N=65 \mathrm{k}, n=6)$.

mas, grain-boundary melting was reported [14. The KTHNY theory was confirmed experimentally for superparamagnetic colloids [15]. Other two-dimensional systems which melt include electrons pinned at a liquid helium interface [16], and surface-adsorbed atomic layers 17.

In this paper, we systematically study two-dimensional melting for repulsive pair interactions using computer simulations. We first concentrate on the inverse powerlaw pair interaction $U(r)=\varepsilon(\sigma / r)^{n}$. This family of potentials includes hard disks of diameter $\sigma$ (for $n \rightarrow \infty$ ) but also, at intermediate $n$, the soft interactions typically found in colloidal particles, and long-range interactions such as dipolar (for $n=3$, 15) and Coulomb forces (for $n=1$, [16]). We will establish that for large enough $n$, the hard-disk melting scenario with its first-order liquidhexatic transition is preserved. Around $n=6$, the system changes over to the classical KTHNY scenario with two continuous transitions. The hexatic phase is firmly established for all parameters studied. To illustrate the gener- 


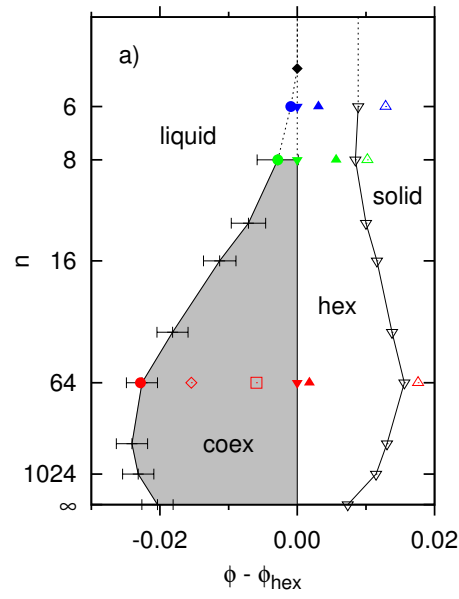

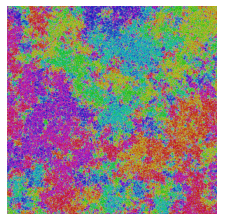

b) $\bullet(8,1.193)$

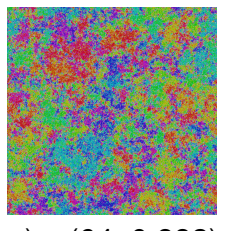

$e) \bullet(64,0.882) f) \diamond(64,0.889)$

c) $\Delta(8,1.201)$
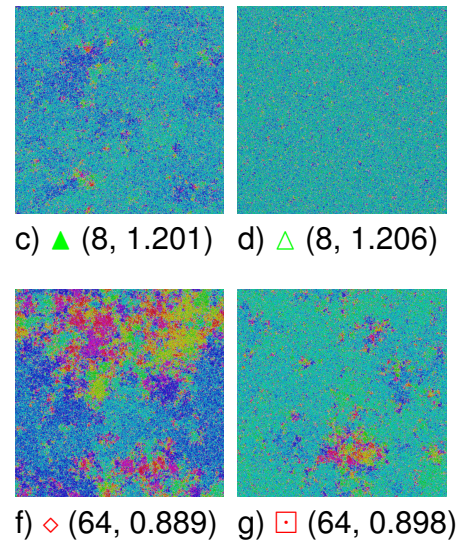
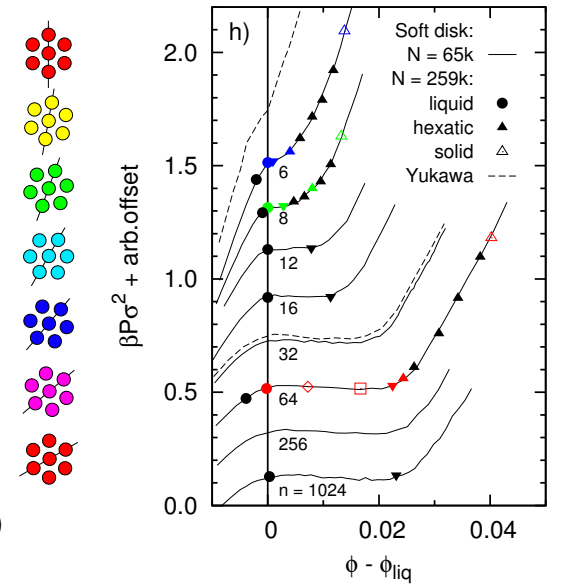

FIG. 2. (Color online) Phase behavior of $r^{-n}$ soft disks for $n \geq 6$. a) Phase diagram as a function of density $\phi$ relative to the density $\phi_{\text {hex }}$ of the pure hexatic at coexistence. The non-monotonic liquid-hexatic coexistence interval vanishes around $n=6$. Symbols match the following figures: bullets are liquid states, filled triangles hexatics, of which downward filled triangles the hexatic at $\phi_{\text {hex }}$; empty triangles are solids. Center: Local orientational order parameter $\psi_{6}$ in $N=259 \mathrm{k}$ particles, the color code is illustrated to the right. Upper row: b) Liquid phase at $(n, \phi)=(8,1.193)$ (subset of a $1036 \mathrm{k}$ configuration) c) hexatic. d) solid. Lower row: Coexistence in the $n=64$ system: e) pure liquid close to coexistence. f) At $\phi=0.889$, the hexatic and the liquid form stripes. g) At $\phi=0.898$, a small bubble of liquid remains on a hexatic background of uniform orientation. h) Equations of state with $n$ from 6 through 1024 (solid lines correspond to $N=65 \mathrm{k}$ soft disks, symbols correspond to $N=259 \mathrm{k}$, $\phi_{\text {liq }}$ is the liquid density at coexistence, dashed lines are for Yukawa particles).

ality of our findings, we approximately map state points of soft disks onto those of particle systems with Yukawa pair interactions by tuning the Debye-Hückel screening length and the temperature. We again identify both the KTHNY and the hard-disk scenarios.

The soft-disk interaction sets no separate energy scale, and we may put $\beta \varepsilon=1$, where $\beta=1 / k_{\mathrm{B}} T$ is the inverse temperature. The phase diagram only depends on the dimensionless density $\phi=\sigma^{2} N / V$, which is related to the dimensionless interaction strength $\Gamma=\beta \varepsilon(\pi \phi)^{n / 2}$. Length scales can be expressed in terms of the interparticle distance $d=(\pi N / V)^{-1 / 2}$. In these units, the pair interaction is $\beta U=\Gamma \times(d / r)^{n}$.

To accommodate the large correlation lengths inherent in two-dimensional melting, we consider systems of $65 \cdot 10^{3}(65 \mathrm{k}), 259 \mathrm{k}$ and $1036 \mathrm{k}$ particles. To equilibrate these large systems, we use Event-Chain Monte Carlo (ECMC) 9], recently parallelized [18] and extended to continuous interactions 19. ECMC displaces a single active particle (red bullet in Fig. 1a) in a fixed direction in successive infinitesimal steps corresponding to a continuous Monte Carlo time. Instead of rejecting a move because of a potential barrier between two particles (dashed line), the displacement is transferred from one particle to the other (Fig. 1b). In this way, cooperative cluster moves are built up. The algorithm is most easily understood for hard-sphere systems, but the concept of pairwise collision events carries over to continuous interactions. ECMC samples the canonical ensemble exactly [19]. It is implemented efficiently using an event-driven approach and mixes faster than con- ventional Monte Carlo [19. Truncating the interaction $\tilde{U}(r)=U\left(\min \left(r, r_{\mathrm{c}}\right)\right)$ at a cutoff distance $r_{\mathrm{c}}$ amounts to neglecting some collision events 24. For soft disks with $n \geq 6$, using the cutoff $r_{\mathrm{c}}=1.8 \sigma$, less than $2 \cdot 10^{-3}$ of collision events are missed (Fig. 1p). ECMC yields the pressure as a zero-cost byproduct of the simulation [19], and allows for the construction of the equation of state. For the cutoff $r_{\mathrm{c}}=1.8 \sigma$, the pressure is reduced by about $1.1 \%$ with respect to the usual cutoff $2.6 \sigma$, but the phase boundaries (e. g., the liquid-hexatic kink in Fig. 1: at $\phi=1.506)$ are not moved, as we checked explicitly.

For each of the $n$ studied, we find extended liquid, hexatic, and solid phases (see Fig. $2 \mathrm{a}-\mathrm{d}$ ). In the liquid, both positional correlations and the correlations of the local orientational order parameter $\psi_{6}$ (with Voronoi weights 22]) are short-ranged, the latter is visualized in Fig. 2 b, e. For large $n$, the equation of state displays a clear Mayer-Wood loop [20] characteristic of a first-order transition (see Fig. 2h; cf. Ref. 9] for a discussion of phase coexistence in the $N V T$ ensemble). At $n=64$, the liquid-hexatic coexistence interval is wider in density than for hard disks $(n=\infty)$, yet qualitatively equivalent: As in Ref. [9, we observe both stripe-shaped coexisting phases (see Fig. 2f), and bubble-shaped minority phases (Fig. 2p). For smaller $n$, the coexistence interval narrows (see Fig. 2a, h) and finally vanishes around $n=6$, where the transition becomes continuous. The computational cost of long-ranged interactions due to larger cutoffs makes large-scale simulations for $n<6$ prohibitively slow.

The phase in coexistence with the liquid is a hex- 

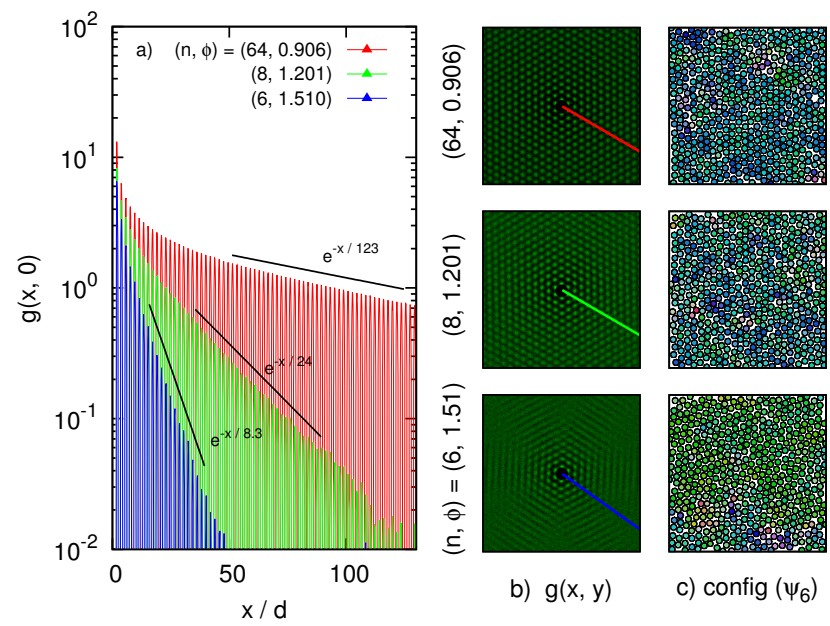

FIG. 3. (Color online) a) Pair correlation function $g(x, y)$ along the $x$ axis in the pure hexatic phase, showing exponential decay for large $x / d$ (ensemble average of $N=259 \mathrm{k}$ configurations after aligning their global orientational order parameters $\Psi_{6}$, as in Ref. 9]). b) Two-dimensional pair correlation function $g(x, y)$ for single configurations at the same parameters. The solid line is the $x$ axis for the left-hand plot. c) Square boxes of side length $40 d$ extracted from the $259 \mathrm{k}$ configurations: Orientational order is preserved as the positional order is lost (color code for $\psi_{6}$ as in Fig. $2 \mathrm{~b}-\mathrm{g}$ ).

atic. Since correlation functions are ambiguous in the coexistence region, we consider pure hexatics above the transition, and find short-ranged positional correlations (Fig. 3a) while orientational correlations are quasi-longranged (see Fig. 4a). The positional correlation length $\xi_{\mathrm{p}}$ can be as large as 100 interparticle distances $d$, but we can equilibrate systems of sufficient size to reveal the asymptotic exponential decay of the ensemble-averaged pair correlation function $g(r) \propto \exp \left(-r / \xi_{\mathrm{p}}\right)$. In the lowest-density pure hexatic, at the transition, $\xi_{\mathrm{p}}$ decreases strongly with $n$ as the coexistence interval vanishes (see Tab. I). At $n=6$, the correlation length is on the order of the interparticle distance $d$. Singleconfiguration pair correlations also confirm that positional order drops from $\xi_{\mathrm{p}} \approx 100 d$ at $n=64$ to a few neighbors at $n=6$ (see Fig. $3 \mathrm{~b}$ ).

We now turn to the analysis of orientational correlations in the transition region. We extract the corresponding correlation length $\xi_{6}$ in the liquid from the asymptotic exponential decay of the correlation function $g_{6}(r)=\left\langle\psi_{6}\left(\boldsymbol{r}_{i}\right) \psi_{6}\left(\boldsymbol{r}_{j}\right) \delta\left(r-r_{i j}\right)\right\rangle$. The orientational correlation length is large but finite in the pure liquid at coexistence. It increases markedly from $n=64$ to $n=8$ and $n=6$ (see the lower three curves in Fig. 4at, data in Tab. I).

At $n=6$, a minute increase in density changes the orientational order from short-range to an algebraic decay with exponent $\approx-\frac{1}{4}$ (Fig. 4 ). This agrees with the KTHNY prediction of orientational correlations $\propto r^{\eta}$ in
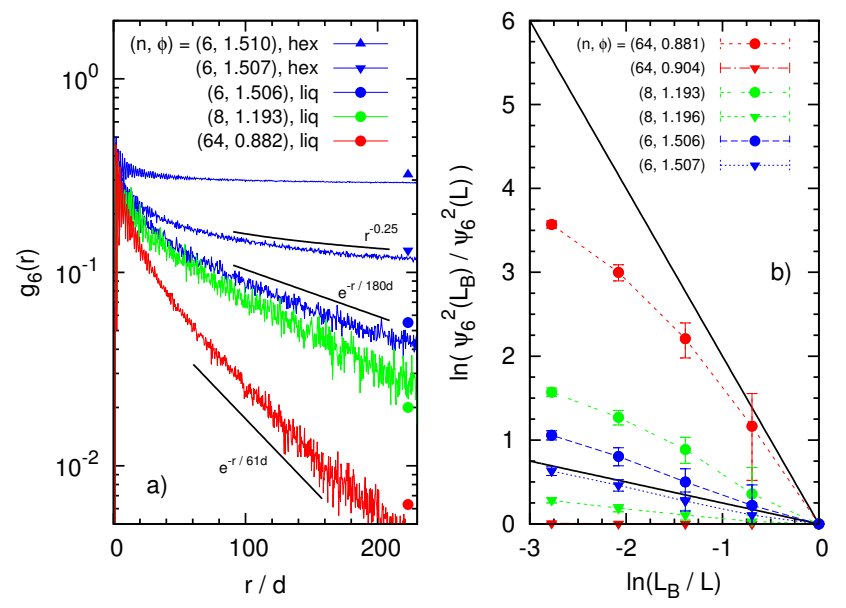

FIG. 4. (Color online) a) Orientational correlations $g_{6}(r)$ close to the liquid-hexatic transition, for several exponents $n$. b) Scaling of the orientational order parameter in subblocks of linear size $L_{\mathrm{B}}$ (see Ref. 21 for details). The KTHNY hexatic is stable below the bold line of slope $-\frac{1}{4}$; short-range order corresponds to the steep bold line of slope -2 . Bullets are liquid states, triangles hexatics (symbols match those in Fig. 2).

the hexatic, with $\eta=-\frac{1}{4}$ at the transition. Away from the transition point, and for $n>6$, the orientational correlation function does not display clear power-law behavior. This is also borne out by the finite-size scaling technique of Ref. 21], computing the average orientational order $\Psi_{6}\left(L_{\mathrm{B}}\right)=\left\langle\psi_{6}\right\rangle_{\mathrm{B}}$ in subblocks of linear size $L_{\mathrm{B}}$. Due to finite-size effects, the liquids at coexistence deviate from the ideal short-range behavior (steep bold line of slope -2 in Fig. $4 \mathrm{~b}$ ), but they are well beyond the KTHNY stability limit for the hexatic (bold line of slope $\left.-\frac{1}{4}\right)$. The $n=64$ and $n=8$ hexatics at the transition have small slopes (Fig. 4p, $\eta=-0.0026$ and -0.10 ), while for $n=6$, we find a value close to the stability limit, $\eta=-0.19$. Our data are thus consistent with a continuous Kosterlitz-Thouless transition for $n \lesssim 6$, which is preempted by a first-order transition for larger $n$.

Approaching the hexatic-solid transition, $\xi_{\mathrm{p}}$ increases, and the ECMC algorithm falls out of equilibrium. Moreover, the effective lattice constant, reduced by a finite equilibrium concentration of defects, is a priori unknown and the positional order in our samples is usually incommensurate with the periodic boundary conditions, leading to frustration effects. This prevents robust conclusions for the exact density of the hexatic-solid transition density $\phi_{\text {hs }}$. Nevertheless, we can provide a lower bound for the melting density $\phi_{\text {hs }}$ from the highest-density configurations that could be molten in a $N=259$ k periodic box (Tab. II). At the hexatic-solid transition, we find no indications of a discontinuity; in particular, the equation of state shows no Mayer-Wood loop (Fig. 2h).

While the correlation lengths in the hexatic are so vastly different in the two regimes, we observe no qual- 

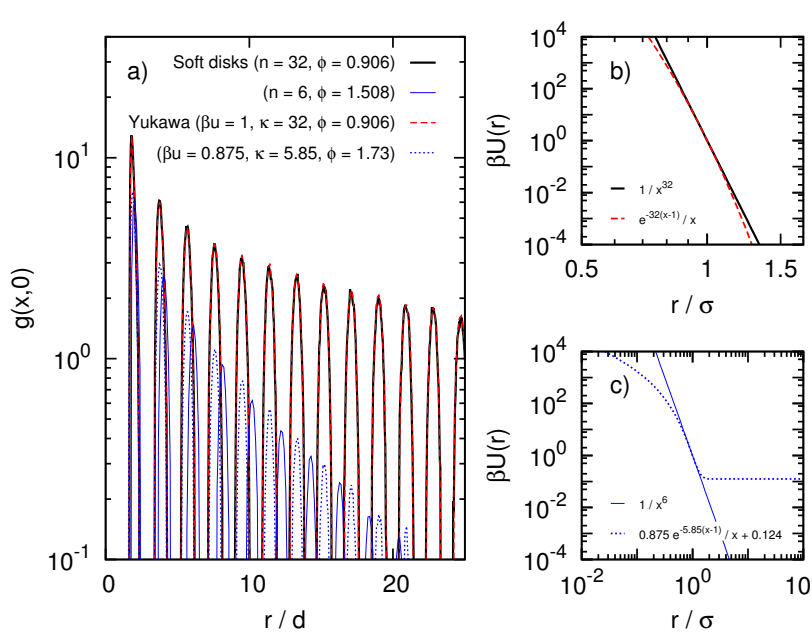

FIG. 5. (Color online) Yukawa interactions exhibiting firstorder (top) and continuous liquid-hexatic transitions. a) Pair correlation function along the $x$ axis, as in Fig. 3a. b, c) Interaction potential of the soft disks and the Yukawa particles around $r=\sigma$.

itative difference in the structure of the KTHNY topological defects, which we define as coordination anomalies in the Voronoi diagram [4, even though this type of analysis is not without problems in disordered systems [22]. The behavior of defects is collective more so than the simple subsequent unbinding picture would suggest. While free dislocations (i. e., isolated pairs of a 5and a 7-coordinated site) exist and free disclinations are indeed heavily suppressed, the majority of defects are involved in more complex clusters. Most defects form stringlike aggregates in which topological charges alternate, and frequently comprise an odd number of bound dislocations. Their classification into grain boundaries, disclinations and dislocations becomes ambiguous. The defect strings circumscribe patches of homogeneous orientational order, and the liquid-hexatic transition can be viewed as the percolation transition of the defect string network. Thus, the liquid-hexatic transition occurs according to a grain-boundary mechanism, similar to proposed direct liquid-solid transitions [6, 7], but starting from a hexatic phase.

The change of scenario from liquid-hexatic coexistence to a continuous transition occurs not only in soft disks as a function of $n$, but whenever the interaction forces can be tuned between the hard-disk and long-range limits. Effectively, only a small part of the interaction potential is explored by the particles: For potentials that, in the relevant range of interparticle distances, are well approximated by a soft-disk potential, the soft-disk phase behavior should be recovered. To test this hypothesis, we use the Yukawa interaction $U(r)=u \times(\sigma / r) \exp (\kappa(1-r / \sigma))$, and match its first and second derivatives at $r=\sigma$ to the respective derivatives of the soft-disk interaction by tun- ing the effective interaction strength $\beta u$ and the DebyeHückel screening length $\kappa^{-1}$ (see Fig. 5b, c). Indeed, we find for the Yukawa system corresponding to soft disks with $n=32$ (parameters $\beta u=1, \kappa=32$ ) a first-order transition (see the lower dashed curve in Fig. 2h) into a hexatic with long positional correlation length $\xi_{\mathrm{p}} \sim 100 d$ (Fig. 5a). Approximating the $n=6$ soft-sphere interaction on the other hand $(\beta u=0.875, \kappa=5.85)$, the transition is clearly continuous (upper dashed curve in Fig. 2h) and the hexatic phase has extremely short positional correlation lengths (Fig. 5 a). These findings agree qualitatively with the results for soft disks. Thus, the change of scenarios identified in this work should be observable experimentally, for example, in charged colloids, planar plasmas, etc., by tuning the Debye-Hückel screening length.

We have shown in this work that two-dimensional melting in particle systems with short-range repulsive pair interactions is generically a two-step transition, with a hexatic phase between the liquid and the solid. We identify two regimes: At large $n$, and for strong screening in the Yukawa particles, we recover the hard-disk melting scenario. In the hexatic phase, at large $n$, positional correlation lengths are two orders of magnitude larger than the interparticle distance $d$. The density of positional defects is correspondingly small. As the interaction potential becomes softer, the nature of the hexatic changes: positional correlation lengths drop to a few $d$, and defects are ubiquitous. The additional entropy due to defects stabilizes the hexatic phase with respect to the liquid state, and the phase-coexistence interval becomes very small. For even smaller $n \lesssim 6$, the liquid-hexatic transition turns continuous, and we recover the standard KTHNY scenario. Conversely, it appears also possible to shift the liquid-hexatic first-order transition towards higher densities. In this case, the hexatic region and the hexatic-solid transition can be preempted by the firstorder transition, giving rise to a direct liquid-solid transition. This has been reported for the "core-softened" potential 23] which includes (at low temperatures) a potential shoulder destabilizing hexagonal order and favoring a direct solid-liquid transition at high density. For large $T$, the core-softened potential of Ref. 23] reduces to the $r^{-14}$ interaction considered here, and would consequently follow a two-step melting scenario with the intermediate hexatic phase. Owing to the long-range nature of interactions, the regime of extremely soft and long-ranged potentials, $n<6$, is not presently accessible to our large-scale simulations, but no further change of scenario is expected for even smaller $n$. Such systems have been considered in experiment [15], and we expect that the change from classical KTHNY two-step melting to a first-order liquid-hexatic transition followed by a Kosterlitz-Thouless-type hexatic-solid transition can be tested experimentally.

This work was supported by a grant of computer 


\begin{tabular}{l|lllll|l}
$n$ & $\beta P_{\mathrm{lh}} \sigma^{2}$ & $\phi_{\text {liq }}$ & $\xi_{6} / d$ & $\phi_{\text {hex }}$ & $\xi_{\mathrm{p}} / d$ & $\phi_{\text {hs }}$ \\
6 & 38.3 & 1.506 & 180 & 1.507 & 2.6 & $>1.516$ \\
8 & 23.1 & 1.193 & 110 & 1.196 & 6.0 & $>1.204$ \\
12 & 14.7 & 0.998 & 112 & 1.005 & 13 & $>1.015$ \\
16 & 12.1 & 0.937 & 95 & 0.949 & 27 & $>0.960$ \\
64 & 9.27 & 0.882 & 61 & 0.904 & 96 & $>0.920$ \\
1024 & 9.17 & 0.889 & 65 & 0.913 & 66 & $>0.924$ \\
$\infty$ & 9.18 & 0.892 & 62 & 0.913 & 51 & $>0.919$
\end{tabular}

TABLE I. Thermodynamic data for $r^{-n}$ soft disks: Pressure $P_{\mathrm{lh}}$ at the liquid-hexatic transition; density $\phi_{\text {liq }}$ and orientational correlation length $\xi_{6}$ of the liquid; density $\phi_{\text {hex }}$ and positional correlation length $\xi_{\mathrm{p}}$ of the hexatic at coexistence. The final column is a lower bound for the melting density (solid-hexatic). The densities are accurate to $\approx 0.5 \%$. Pressures are computed using the truncated interaction and are thus low by at most $1.1 \%$. The statistical sampling error is a decade smaller (error bars in Fig. 1f). The correlation lengths are determined from the tail of the respective correlation functions and are subject to large errors of $\pm 10 \%$ due to the choice of $\phi$. They are consistent with earlier results for hard disks 9.

time at the PSL Computing Centre MesoPSL. We thank H. Kleinert, O. Dauchot, and the referees for insightful comments.

* sebastian.kapfer@fau.de

† werner.krauth@ens.fr

[1] B. J. Alder and T. E. Wainwright, Phys. Rev. 127, 359 (1962).

[2] N. D. Mermin and H. Wagner, Phys. Rev. Lett. 17, 1133 (1966).

[3] B. I. Halperin and D. R. Nelson, Phys. Rev. Lett. 41, 121 (1978); D. R. Nelson and B. I. Halperin, Phys. Rev. B 19, 2457 (1979); A. P. Young, Phys. Rev. B 19, 1855 (1979).

[4] K. J. Strandburg, Rev. Mod. Phys. 60, 161 (1988).

[5] C. Alba-Simionesco et al., J. Phys.: Condens. Matter 18, R15 (2006).

[6] S. T. Chui, Phys. Rev. B 28, 178 (1983). Y. Saito, Phys. Rev. B 26, 6239 (1982).

[7] H. Kleinert, Stresses and Defects: Differential Geometry, Crystal Melting, Vol. II of Gauge Fields in Condensed Matter (World Scientific, Singapore, 1989). http://www. physik.fu-berlin.de/ kleinert/re.html\#b2

[8] Y. Lansac, M. A. Glaser, and N. A. Clark, Phys. Rev. E 73041501 (2006).

[9] E. P. Bernard and W. Krauth, Phys. Rev. Lett. 107, 155704 (2011).

[10] T. Franosch, S. Lang, and R. Schilling, Phys. Rev. Lett. 109240601 (2012); W. Qi, A. P. Gantapara, M. Dijkstra, Soft Matter 10, 5449 (2014).

[11] Wei-Kai Qi, Shao-Meng Qin, Xiao-Ying Zhao, and Yong Chen, J. Phys. Condens. Matt. 20, 245102 (2008).

[12] A. H. Marcus and S. A. Rice, Phys. Rev. Lett. 77, 2577 (1996).

[13] Bo-Jiun Lin and Li-Jen Chen, J. Chem. Phys. 126, 034706 (2007).

[14] V. Nosenko, S. K. Zhdanov, A. V. Ivlev, C. A. Knapek, and G. E. Morfill, Phys. Rev. Lett. 103, 015001 (2009).

[15] K. Zahn, R. Lenke, and G. Maret, Phys. Rev. Lett. 82, 2721 (1999). U. Gasser, C. Eisenmann, G. Maret, and P. Keim, Chem. Phys. Chem. 11, 963 (2010).

[16] C. C. Grimes and G. Adams, Phys. Rev. Lett. 42, 795 (1979).

[17] P. A. Heiney, P. W. Stephens, R. J. Birgeneau, P. M. Horn, and D. E. Moncton, Phys. Rev. B 28 6416 (1983). E. D. Specht, M. Sutton, R. J. Birgeneau, D. E. Moncton and P. M. Horn, Phys. Rev. B. 301589 (1984).

[18] S. C. Kapfer and W. Krauth, J. Phys. Conf. Ser. 454, 012031 (2013); arXiv:1301.4901.

[19] M. Michel, S. C. Kapfer, and W. Krauth, J. Chem. Phys. 140, 054116 (2014); arXiv:1309.7748

[20] J. E. Mayer and W. W. Wood, J. Chem. Phys. 42, 4268 (1965).

[21] K. Bagchi, H. C. Andersen, and W. Swope, Phys. Rev. Lett. 76, 255 (1996).

[22] W. Mickel, S. C. Kapfer, G. E. Schröder-Turk, and K. Mecke, J. Chem. Phys. 138, 044501 (2013); arXiv: 1209.6180

[23] D. E. Dudalov, Y. D. Fomin, E. N. Tsiok, and V. N. Ryzhov, Phys. Rev. Lett. 112, 157803 (2014); D. E. Dudalov, E. N. Tsiok, Y. D. Fomin, and V. N. Ryzhov, J. Chem. Phys. 141, 18C522 (2014).

[24] ECMC, in contrast to standard Monte Carlo, acts on the pairwise and not on the total interaction energy (factorized Metropolis filter [19]). Techniques such as the Ewald summation are not required even for $n<6$ : The pairwise formulation permits us to include long-range interactions outside of the cutoff in a probabilistic way (not required for $n \geq 6$ ). 